\title{
Del Sistema al Prototipo y viceversa (el caso de la GBH + INVISO)
}

\section{From System to Prototype and vice versa (GBH case + INVISO competition)}

\author{
J. Guardiola Arnanz ${ }^{(*)}$, J.M. Reyes González ${ }^{(* *)}$
}

\section{RESUMEN}

La mayoría de las viviendas industriales famosas del SXX no son solo eso (prototipos individuales): forman parte de un sistema general de grado muy superior para ponerlo a prueba. Se demuestra en este artículo como el recorrido en esta clase de concepción productiva no se efectúa "desde el prototipo al sistema" por una simple adición del modelo; al contrario, estos prototipos resultan del camino inverso, para luego sí, servir como feed-back.

Se muestran 5 antecedentes históricos dentro de este recorrido, y se analizan más a fondo el Primer premio del concurso INVIso y la casa GBH. De su estudio se derivan algunas conclusiones en cuanto a la relación entre la Teoría General de Sistemas y las premisas que la Industrialización Abierta confiere a los Sistemas construidos a partir de componentes compatibles (S.3c), como la flexibilidad, o el reciclaje, y la manera de conseguir que estas se cumplan mediante procedimientos probados.

Palabras clave: Teoría General de Sistemas, prototipo, industrialización abierta, componente, procesos.

\section{ABSTRACT}

Main subject of this article is showing how most of famous manufacturing houses of XX Century are not only single prototypes, but they really are part of general system from upper level (system they want testing). Also, we can prove how this kind of generation don't running "from Prototype to the System" (by single juxtaposition of the model to increase scale). On contrary way this prototypes are the result of inverse procedure (after, they will be as feed-back to refine start system).

To this purpose the article show 5 historic cases as background within this behavior line, and INVISO winner and GBHouse are analyze most deep. From this studio we can get some conclusion about relation between "General Theory of Systems" and "open industrialization" premises give to S.3c (System constructed by compatibles components): as flexibility or recycling, and how we can get it through proving procedures.

Keywords: General Theory of Systems, prototype, open industrialization, component, process.

(*) Ing. Industrial y Arquitecto, Prof. en ICAI y miembro de AECMA (Madrid).

(**) Dr. Arquitecto, Prof. en Universidad Alfonso X el Sabio (Madrid), y de Postgrado en ETSAM y TUWien. Persona de contacto/Corresponding author: reyesjm.arq@gmail.com (J.M. Reyes González). ORCID: http://orcid.org/oooo-0002-3892-0254 (J. Guardiola Arnanz); http://orcid.org/oooo-0003-4293-5480 (J.M. Reyes González).

Cómo citar este artículo/Citation: Guardiola Arnanz, J.; Reyes González, J.M. (2019). Del Sistema al Prototipo y viceversa (el caso de la GBH + INVISO). Informes de la Construcción, 71(555): e299. https://doi.org/10.3989/ic.61889.

Copyright: (C) 2019 CSIC. Este es un artículo de acceso abierto distribuido bajo los términos de la licencia de uso y distribución Creative Commons Reconocimiento 4.o Internacional (CC BY 4.0). 


\section{INTRODUCCIÓN \\ (Relación de las partes con el todo, y las partes entre sí)}

Contrariamente a lo que se cree en bastantes círculos profesionales y académicos (en el campo de la industrialización de la construcción) el prototipo no suele ser el experimento del que se parte y que después da lugar a un sistema espacial cualquiera a partir de sus componentes. Por el contrario, tras del prototipo suele existir toda una teoría espacial e incluso urbana que es la que le dota de valor, para dejar de tener sentido como un hecho aislado más o menos acertado.

En la producción de naves y torres esto sí está asumido, pero no suele ser así al referirnos a la vivienda en serie. En este campo parece que la unidad a repetir debe ser la propia vivienda, y que su mera adición garantiza cualquier agrupación valida. Así, se cree que una vez solucionado el prototipo se tiene solucionada cualquier agrupación de viviendas. Nada más lejos de la realidad aceptable (esto solo es válido al referirnos a la vivienda exenta, donde la clase de agrupación se remite a la ordenación urbana).

El repaso por los más celebres prototipos de vivienda industrializada del Siglo XX sirve para comprobar las afirmaciones anteriores. Y el Primer Premio del concurso INVISO, y la casa GuardiolaBabeckaHouse (GBH), sirven para comparar resultados, motivos, e intenciones desde una experiencia más cercana y conocida.

Todo ello pone de manifiesto la importancia del prototipo en el $\mathrm{I}+\mathrm{D}+\mathrm{i}$ dentro de la industrialización de la vivienda, y que no sean valorados equivocadamente como hechos individuales completos y acabados dentro de la trayectoria profesional de sus autores.

El conocido criterio Académico que define la belleza como "la necesaria relación armónica de las partes con el todo, y las partes entre sí”, aquí se debe aplicar entre el sistema general y el prototipo (no considerar el prototipo como un todo autónomo y acabado en sí mismo: un sistema cerrado).

\section{ANTECEDENTES DESTACADOS}

Arrancado al comienzo del Racionalismo en S. XX (y prescindiendo de la Ballom-Frame, ya muy desarrollada anteriormente), los primeros manifiestos de la vivienda en serie pueden situarse en la casa Domino (1914), casa Citroän (1917), y el Sprite Nouveau (1924) (1), todas propuestas por Le Corbusier (LC). Sin embargo, en su pertenencia a un sistema de orden superior, contradicen las premisas del epígrafe anterior: el prototipo es la unidad que se repite como un módulo espacial completo, y vienen a configurar: topologías urbanas (Domino), manzanas (Sprite Nouveau), y vivienda aislada (Citroän). Pero entonces la repetición y la uniformidad se entendían como la solución adecuada en su situación política internacional.

Sin embargo, si cruzamos el Atlántico, R.B. Fuller en USA y su Dymaxion (1927-43) (Figura 1a), la solución tecnológica va más allá que del hormigón armado de LC. Los medios de producción cercanos permiten concebir una edificación muy ligera y eficaz (entonces se pasa de la Escuela de Sullivan en Chicago al Crysler en NYC, y Neutra construye la primera vivienda en acero del continente). La Dymaxion es desde entonces el ejemplo por antonomasia de vivienda industrializada (como hito de partida), pero se olvida que su inventor, además de querer construir el prototipo que todos conocemos y producirlo en serie, también proponía en paralelo el " $4 \mathrm{D}$ timelock" (Figura 1b): un sistema universal de torres de 10 a 15 pisos de altura que definían asentamientos conectados entre sí a través de un pequeño vehículo hibrido capaz de volar y rodar sucesivamente (dymaxion-car). Estas torres se componen de una sucesión de pisos que son la propia dymaxion superpuesta en vertical. En este momento la notable ineficacia térmica del prototipo aislado deja de lastrarlo, y la flexibilidad espacial que anuncia se remultiplica al comenzar a aparecer toda clase de usos mezclados con el residencial (gimnasios, lavanderías, talleres, etc...) (2). Torres que desembocaron en los célebres silos de la "plug-in city" (archigram, 1967).

Avanzando 20 años más por la misma geografía, la Eames House (L.A.Ca. 1946-49) (Figura 2a) no consiste solo en

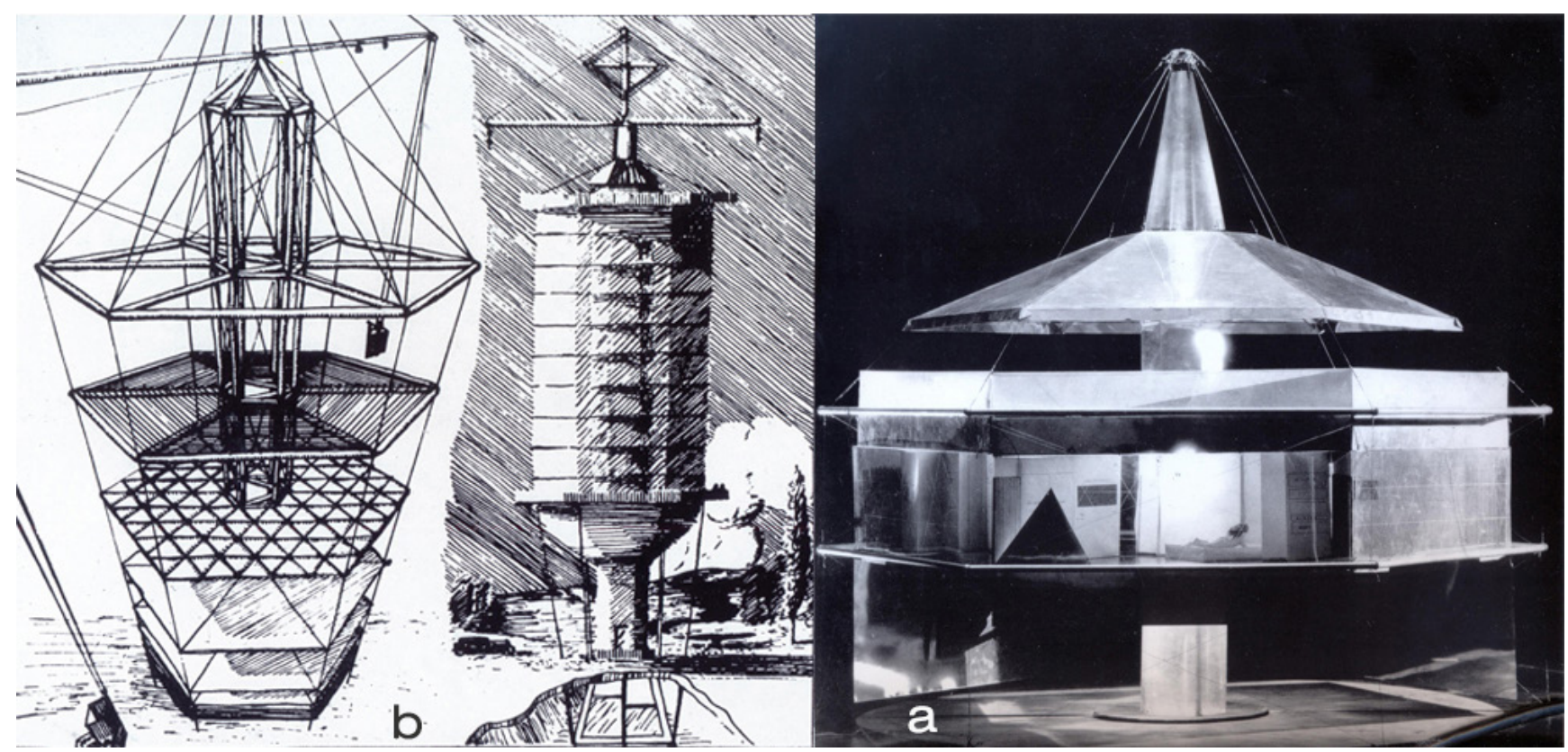

Figura 1. a) casa Dymaxion; b) 4D timelock 


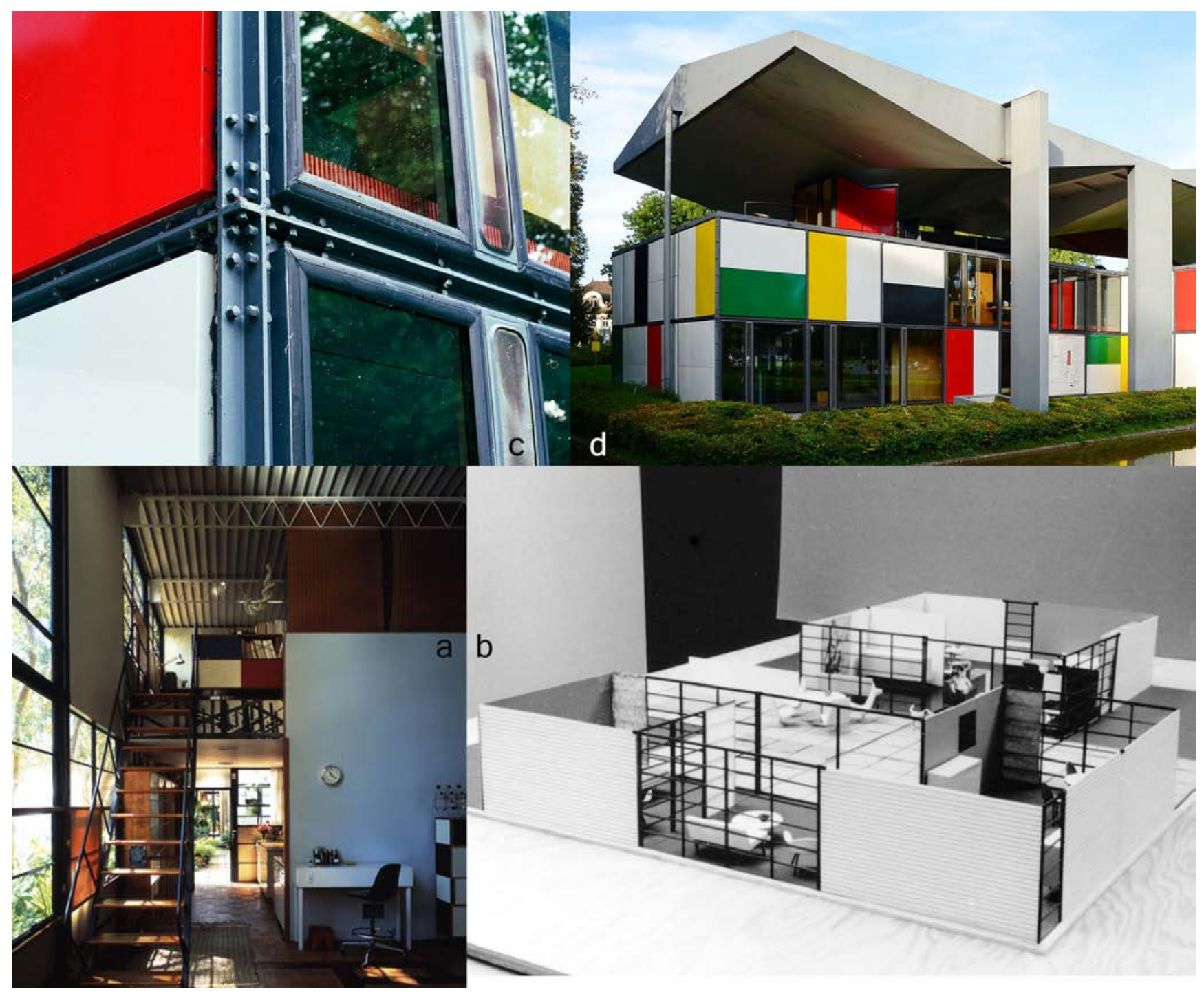

Figura 2. a) interior Eames House; b) The Rewell Toy House; c) sistema 226x226x226; d) Fundación Heidi Weber.

sus 2 celebres paralelepípedos capaces de conformar patios, porches, y veredas en su entorno inmediato: responde a un sistema espacial más complejo denominado "The Revell Toy House" (Figura 2b) que sirve para conformar una agrupación más general respetando esta clase de espacios exteriores. Sistema que también se usó en la edificación del showroom para la compañía de muebles de Herman Miller en L.A (3).

"La Casa del Hombre" (LC, Zurich 1967), actual Fundación Hiedi Weber) (Figura 2d), está basada en el desarrollo del sistema 226x226x226 (Figura 2c) (1). Esta obra parece conciliar al Arquitecto con la versión de la Casa Citroän que Eames consigue materializar valiéndose de la tecnología USA de posguerra. El 226x226x226 se proyecta en 1948 (mientras Eames corregía la primera versión de su casa por la Citroän), y para aplicarlo en el grupo de apartamentos de vacaciones "rot et qob" en Cap Martin. (un L.C. que en aquellas fechas se resiste a abandonar el acero por el H.A., y que no lo consigue recuperar hasta sus últimos días).

En el mismo escenario tecnológico que Eames, y tras otros 20 años, E. Schulitz pone en pie la casa TEST (L.A.Ca.1972) (Figura 3a), otro importante prototipo en la historia de la industrialización ligera residencial, que implica un sistema de agrupación capaz de admitir infinidad de combinaciones y variaciones espaciales y materiales para adaptarse a distintas necesidades de sus habitantes (Figura 3b) (siempre dentro de la estandarización que la producción industrial necesita) (4).

En la misma década, R. Piano nos deja su Abiazioni Evolutiva (Cusago 1977) (Figura 3c), un prototipo donde se cambia el acero americano por el H.A. europeo, pero sin renunciar a las ventajas de la Industrialización ligera (entrepisos y carpinterías son de madera, y las vigas metálicas) (5). Como en el caso anterior, el sistema general de agrupación se acopla a las curvas de nivel pero ahora en la cuenca Mediterránea, genuino territorio del TEAM X donde reivindicar los patios, porches y terrazas que esta clase de arquitectura estandarizada, pero variable, practica con total decisión y firmeza (Figura 3d).

Ya en el III Milenio, nombrar el System-3 de KFN (MoMA 2008) (6), SML home (SDecathlon-europe, UCH 2010) (7), o la Casa Garoza (Herreros, Ávila 2014) (8). Todos ellos prototipos que son solo la materialización parcial de sus sistemas de agrupación respectivos), y que ya emplean las unidades $3 \mathrm{D}$ como los componentes de construcción más propios para el S.21. 


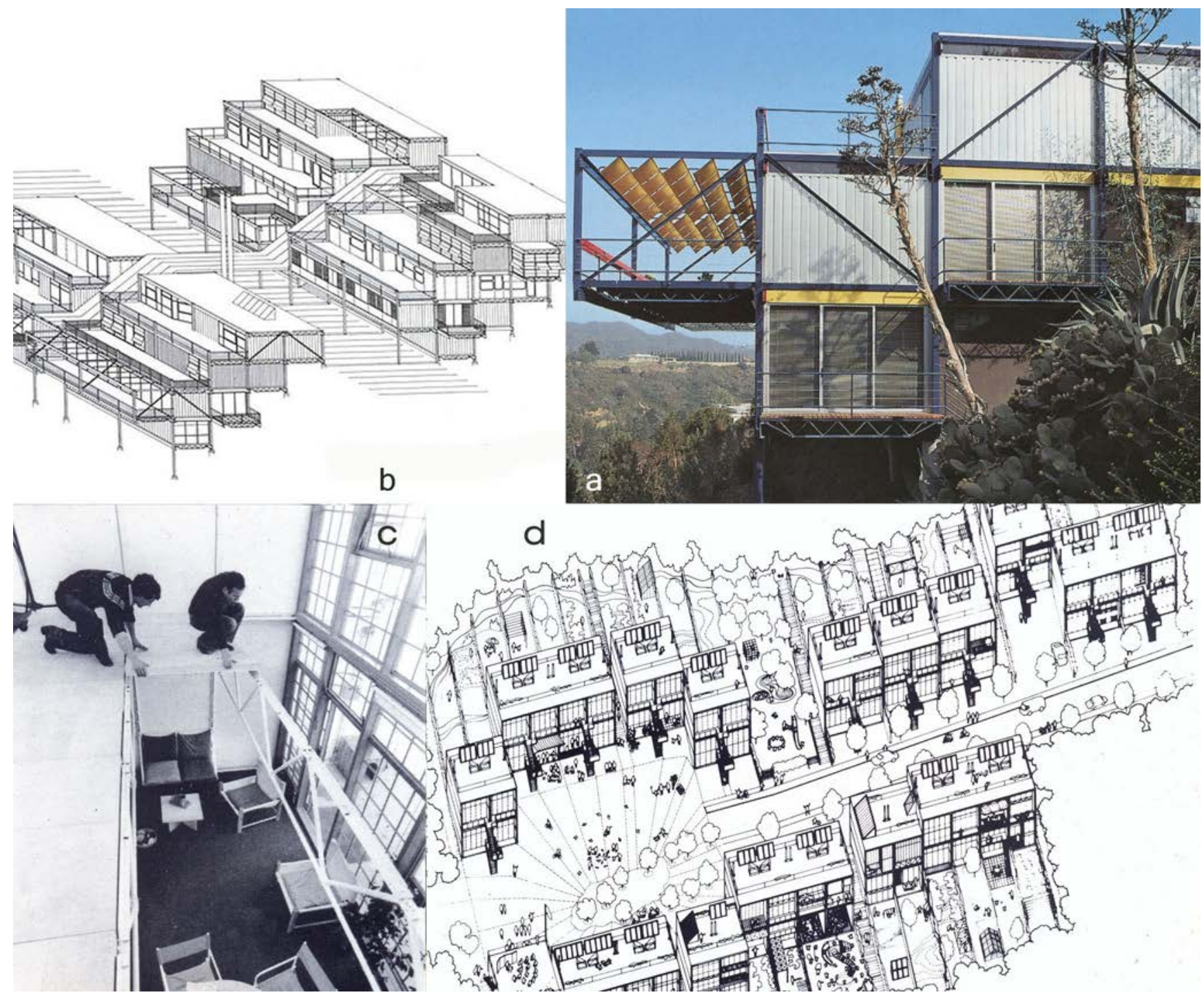

Figura 3. a) casa TEST; b) TEST system; c) interior abitazioni evolutiva; d) Ab. Evolutiva urban.

3. INVISO Industrialización de la Vivienda Sostenible (1er. Premio en concurso de ideas EMVs. Arquitectos: Guridi+Tartás; J.Guardiola; A.Garcia)

Esta propuesta surge a partir de la convocatoria por parte del grupo de investigación INVISO de un concurso de ideas. El objetivo del mismo fue la mejora de las técnicas y procedimientos de construcción para aumentar la calidad del producto final, así como la funcionalidad y mantenimiento de la vivienda. Y la respuesta dada por el equipo ganador es guiarse por los principios de la "industrialización-abierta", y crear así un S.3c capaz de generar una agrupación de viviendas que, siendo estandarizadas, puedan además adaptarse a ciertas necesidades particulares de sus habitantes (a través de variaciones y posibilidades que el propio sistema proporciona automáticamente): flexibles (el enunciado del concurso no fija situación urbana real, ni normativa a la que ceñirse).

Así, se plantea una agrupación de viviendas desarrollada en bloque lineal en altura con viviendas abiertas directamente a las dos orientaciones exteriores opuestas. Dada la sostenibilidad energética exigida en las bases del concurso, se favorece más una de las orientaciones respecto a la captación solar, frente a su opuesta que pasa a estar más protegida (cerrada) por ser preferentemente la Norte, al no disponer de normativa ni parcela alguna que condicione esto.

Con estas determinaciones previas, la planta general de la agrupación queda distribuida como una "topología espacial en peine" (9) con 2 bandas longitudinales (B1 y B2) +1 Zona central (Zc) entre ambas + 2 zonas de expansión (Ze), en orientaciones Norte y Sur (Figura 4). En esta clase de esquema topológico, una de las 2 bandas se especializa mucho. En este caso, la B2 acoge todos los espacios asociados a instalaciones y zonas húmedas, mientras la B1 queda libre y como espacio complementario a su correspondiente en B2 (relación directa entre espacio servidor y espacio servido). Entre B1 y B2 se determina una Zc que funciona como zona de transición entre ambas, que se ocupa por circulaciones o espacios complementarios a las bandas (sirven para que las viviendas funcionen sin conflicto alguno: espacios compatibles entre sí y con los colindantes). La Ze Norte sirve para situar las galerías de acceso y las redes generales de suministro, y la Ze Sur para colgar balcones, toldos, captadores solares, o depósitos de agua, allí donde estos componentes sean necesarios. 


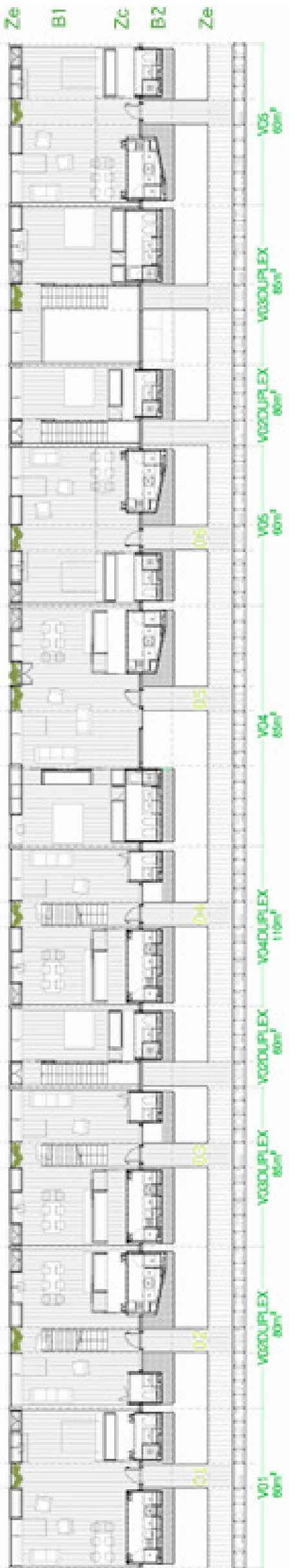

Figura 4. Sistema INVISO: planta general.
Fijada esta distribución general, sus dimensiones y modulación, se obtiene la tipología de edificación, y se pueden insertar en la misma los componentes materiales del sistema sin conflicto alguno.

- Estructura: estructura reticular de acero a base de vigas y pilares con nudos atornillados, con unas luces máximas de 4,80 m. (Figura 5a). Los pisos son tableros de madera reforzados con perfilería metálica a base de correas inferiores o paneles rizados. La cualidad material de todos estos componentes hace que el mecano sea muy fácil de desmontar y modificar (construcción en seco), pudiendo extenderse donde se necesite, o generar dobles alturas donde se desee (se producen viviendas de 1 y 2 alturas).

- Cerramiento Sur: a base de paneles sándwich ligeros, y carpintería metálica para vidrio aislante.

La retícula estructural de acero sirve como BUS para colgar la piel continua allí donde se necesite, y además, también permite conectar a la misma toda clase de complementos espaciales para conseguir una Zona de expansión (Ze) que funciona como filtro regulador de energía (balcón, toldos, depósitos, paneles FV, etc. (Figura 5a).

- Cerramiento Norte: se reserva para colgar las unidades $3 \mathrm{D}$-compact que contienen los "mecanismos y aparatos húmedos" necesarios para que la banda B1 se encuentre correctamente servida según sea la variación de vivienda a que correspondan. Con estas unidades $3 \mathrm{D}$, más el puente de entrada a las viviendas, todo el cerramiento de esta vertiente queda cubierto y hermético. Una grúa guiada desde la cubierta, permite cambiar de sitio o reponer los $3 \mathrm{D}$ comp con total facilidad. El cerramiento de las unidades es un monocasco metálico registrable que actúa a la vez como estructura y corteza aislante.

- Instalaciones: su disposición y organización son fundamentales para el buen funcionamiento de la propuesta. Toda la cara Norte se reserva para el paso de las mismas, pasan entre la galería de acceso y la retícula estructural de acero, y una vez colgadas de esta, y siempre a la vista, sirven como un BUS de sistema al cual poder aplicar en sus puntos de conexión cualquiera de las unidades $3 \mathrm{D}$ que el catalogo pone a disposición de los usuarios. Con 3 clases de baño y 3 de cocina, se consiguen todas las variaciones que aparecen en la planta general (Figuras 4 y $5 \mathrm{c}$ ).

Todos los circuitos necesarios en la $\mathrm{B} 1$ son vistos y registrables (en bandejas colgadas), y salen de la B2; aunque prevalece el control wire-less de todos los aparatos y luminarias (consultar (10) para ampliar información). Se define así un S.3c con componentes autónomos, intercambiables, y sostenible por su descomponibilidad.

\section{GBH GuardiolaBabeckaHouse, Madrid (Arquitectos:Guridi+Tartas y JGuardiola + A.García)}

Se sitúa en un solar entre medianeras con un anchura de $9 \mathrm{~m}$ y una profundidad de edificación de $11 \mathrm{~m}$. El programa es para 2 viviendas independientes (una en el ático, y otra en el resto con sótano y garaje incluido). El programa de usos espaciales se reparte como en el caso anterior según un esquema de "organización en peine", pero ahora son los costados Este y Oeste los que dan al exterior, mientras los N y S quedan ciegos (son las medianeras).

B2 acoge los recintos más privados (aseos+ dormitorios + despacho), mientras la B1, contiene los espacios más co- 

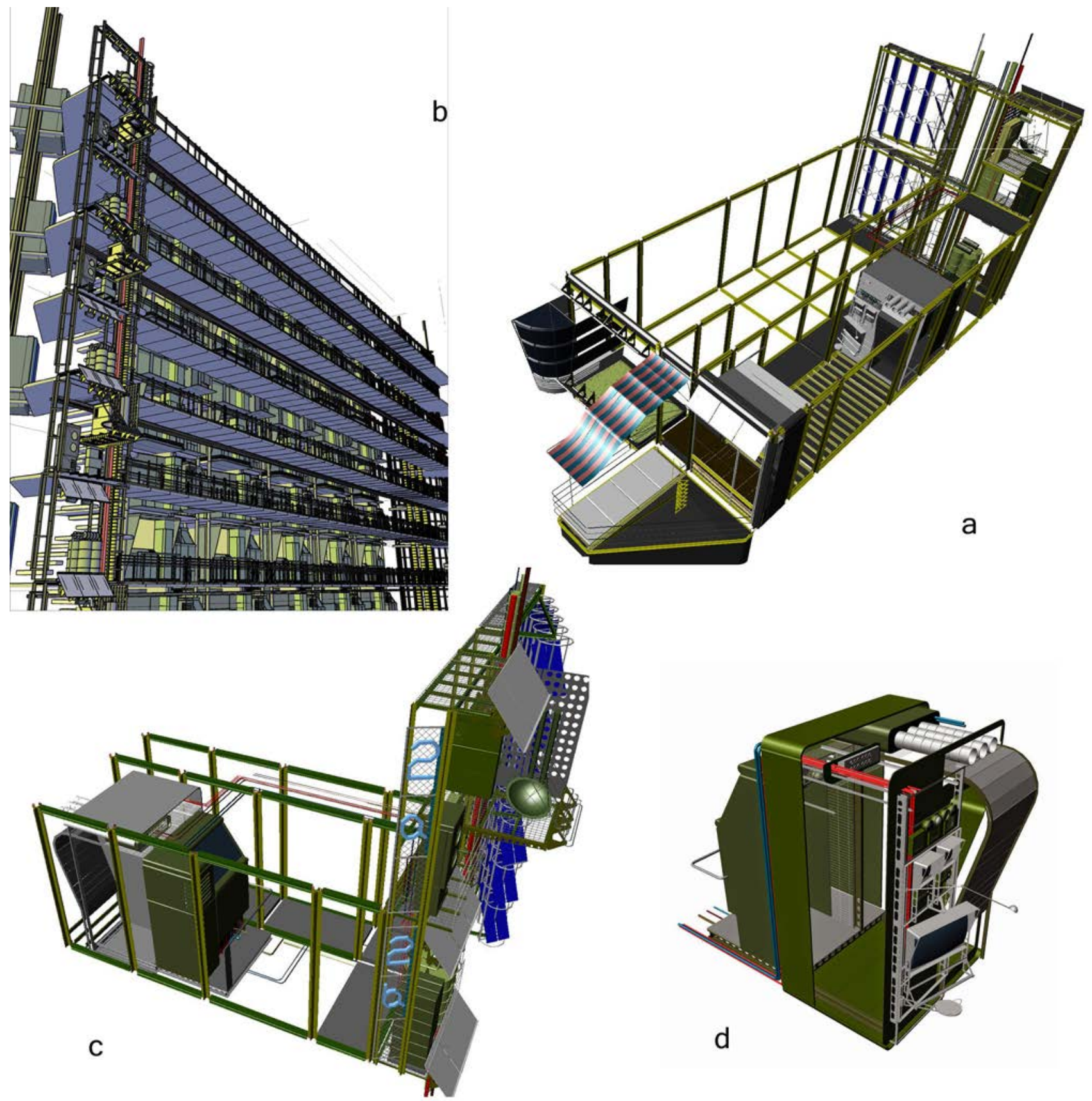

Figura 5. a) INVISO: retícula+ componentes; b) cara Norte; c) BUS para instalaciones; d) unidad 3D-compact.

munes (salones, taller, garaje, y comedor con cocina). La Zc (zona de transición en esta topología) se ocupa por los fluidos: escalera+ascensor+montante vertical de instalaciones (Figura 6). Las Ze no se consideran, solo un retranqueo en el ático sirve como filtro regulador a esta parte del edificio.

En B1 y B2, los pisos se contrapean con distinta cota según la escalera de un solo tiro reparte las mesetas donde se necesitan para acceder a las estancias de B2. La flexibilidad en B2 se ve condicionada por sus accesos, pero permite que las salas en $\mathrm{B} 1$ disfruten de unas alturas considerables.

- Estructura: las medianeras son pantallas de hormigón portante porque el aislamiento contra el fuego condiciona mucho el sentido de los pórticos (ortogonales a la calle). A estas dos se añade otra más entre B2 y Zc para arriostrar el sistema. Así, queda un solo pórtico metálico entre Zc y B1), suficiente para poder ensayar la estructura reticular del sistema INVISO.

Los pilares convexos en cruz son 4 PNL 100.100 que abrazan en sus capiteles las cartelas atornilladas que reciben a su vez las vigas convexas conformadas con 2 PNC.280 (Figura 7a). Los pisos son de dos clases: garaje y separación con ático, y resto.

Los primeros son de paneles de chapa rizada colaborante, y los segundos de tablero aglomerado de madera sobre viguetas de chapa plegada (2C.140) (Figura 7b). El pavimento es de tarima enrrastrelada de madera natural.

- Cerramiento: mediante el mismo sistema de conexión a la retícula estructural, se plantean 2 soluciones materiales muy distintas a Este y Oeste. La primera muy cerrada al sol poniente y la calle (Figura 8a), y la segunda muy traslúcida al sol naciente y el patio propio. 

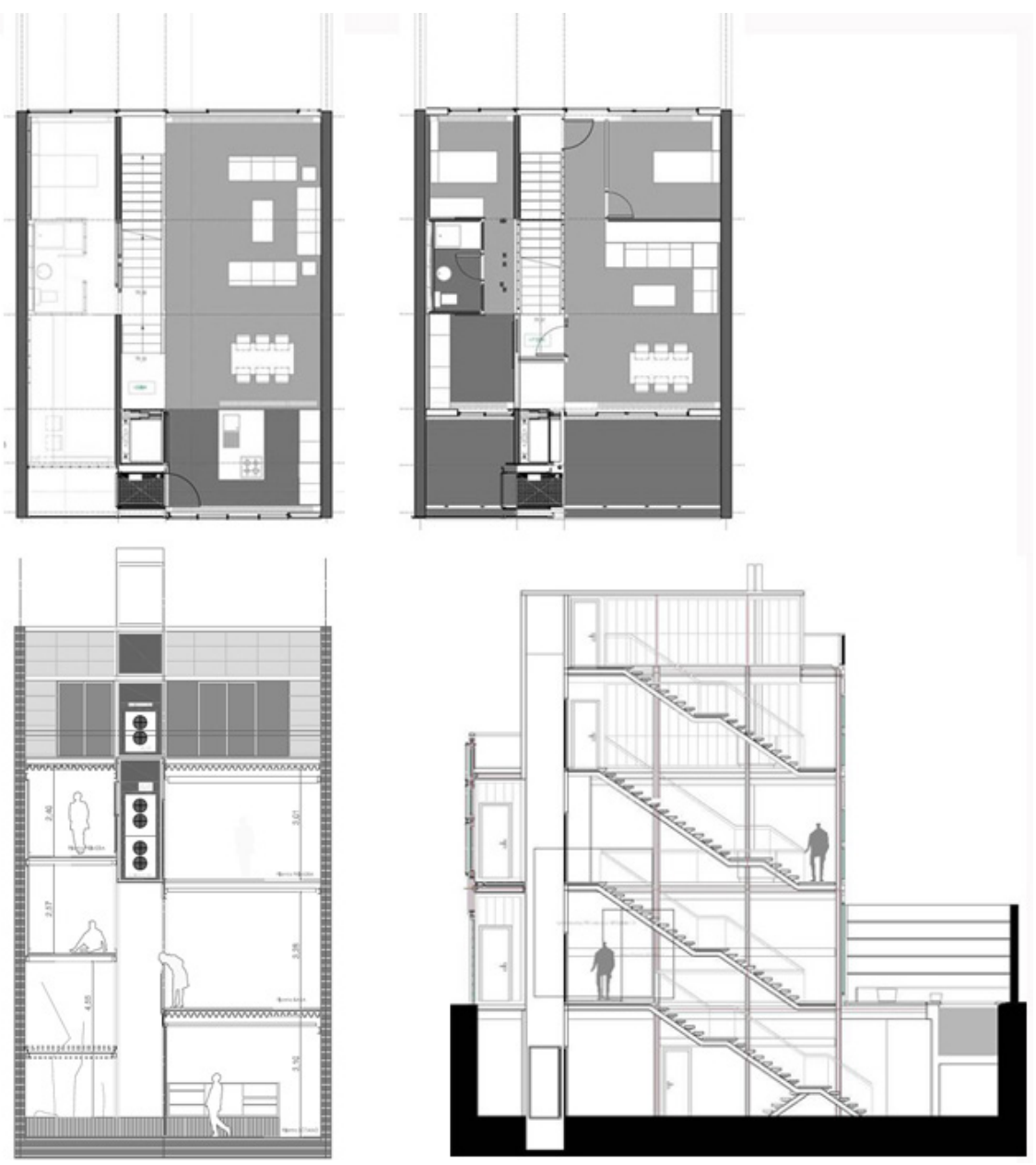

Figura 6. GBH: planta general y ático + secciones.

El cerramiento Oeste es multicapa, siempre por delante de los zunchos de piso. Todas las hojas del sistema quedan sujetas a los principales montantes verticales a través de sus líneas de anclaje horizontal en las cotas correspondientes a alféizar y dintel metálicos (Figura 8b) (que definen los huecos horizontales donde encajar la carpintería). El cerramiento y su instalación son independientes de la estructura de los pisos y protegen el puente térmico. La hoja exterior es de placa "composite" opaca, y la interior de cartón-yeso. El núcleo aislante separado de las placas, configura un cerramiento transventilado. El vidrio queda protegido al exterior por una celosía deslizante de "deploye", y la composición del alzado conlleva una lectura directa de su sistema constructivo (la Zc donde se producen las circulaciones y suministros del sistema aparece como un paño vertical sin interrupción vertical alguna (Figura 8a).

En la vertiente Este, se sustituye el sándwich opaco por paneles de U-glass traslucido allí donde se busca mayor iluminación, y su sistema de anclaje a la retícula base sigue siendo el mismo que en la vertiente oeste (2 líneas horizontales de apoyo) (Figuras 8c y 8d).

- Instalaciones: EL mazo principal de circuitos verticales se aloja en Zc con todos sus cuadros de registro al exterior. De esta Zc se reparten por las B1 y B2 las bandejas necesarias con todo el cableado para el suministro puntual de energía. Todos los circuitos son vistos y registrables (Figura 9). Los aseos situados en B2 ocupan una situación central o extre- ma en cada planta según se distribuyen camas y zonas de trabajo. El suministro y desagüe de los mismos se realiza muy fácilmente debido a lo anterior.

La calefacción es a base de aire caliente expulsado por los bordes inferiores de cada piso junto al cerramiento exterior, y su instalación es independiente de la estructura y el cerramiento (Figura 8b).

El principio de accesibilidad de las instalaciones, canalizaciones, y equipos (su registro), que de forma tan explícita está considerado en el INVIso se ensaya así en la GBH.

- Divisiones interiores: se utilizan al mínimo estos componentes: solo separan aseos de sus espacios colindantes, y garaje de recibidor (Figura 9) (la pantalla de arriostramiento interior separa directamente B2 del resto).

Las mamparas de aglomerado y perfilería interior metálica tienen acabado de melanina en colores primarios. Para evitar la transmisión acústica, en las juntas de sus apoyos inferior y superior se corta el pavimento y se coloca un techo en los aseos.

El resultado total son unas amplias y luminosas estancias donde su sistema constructivo se lee explícitamente en cualquier punto, y sirve para componer directamente su definición estética (Arquitectura Brutalista). Una arquitectura que permite actuar sobre su sistema constructivo inmediatamente y sin error, para cambiar, sustituir, o modificar cualquiera de sus piezas (Figura 10). 

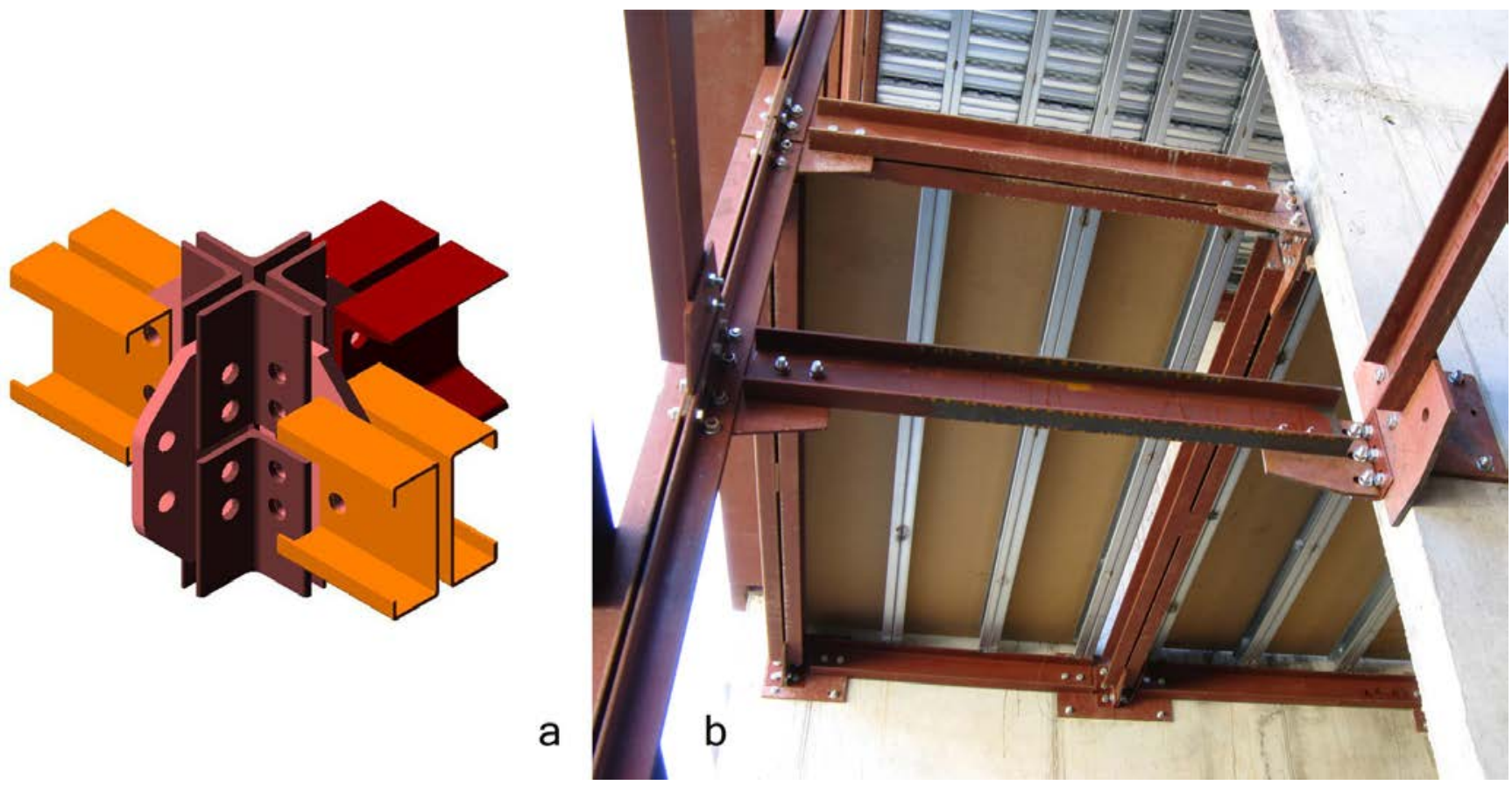

Figura 7. a) nudo general; b) pisos de chapa y tablero.

\section{ANÁLISIS SISTÉMICO DE CASOS}

\subsection{Flexibilidad y construcción en seco}

Los casos presentados se construyen a partir de materiales ligeros, y su ensamble se produce mediante "construcción en seco". Esta construcción favorece los procesos tanto de montaje como desmantelamiento (disassembly) del sistema que generan. Esto favorece el intercambio o sustitución de piezas sin crear destrozo alguno en el resto del sistema.

El hecho de que esto sea posible, no implica que el sistema sea flexible en sí. Un "sistema-cerrado" es aquel producido por un mismo fabricante que no admite el intercambio o sustitución de piezas provenientes de otras marcas. Cuando sucede lo contrario, estamos ante un sistema producido mediante "industrialización-abierta", y estos si son sistemas flexibles.

Según este criterio: la Dymaxion y la Abitazioni Evolutiva son sistemas-cerrados, mientras el resto pertenecen a sistemas-abiertos resueltos mediante "industrializaciónabierta”. La Eames-H utiliza un esqueleto estructural a partir de componentes de catálogo ya existentes previamente para la construcción de naves industriales (vigas en celosía y pilares en tubo de acero normalizados + ventanas basculantes estandarizadas. Los tableros de madera para sus cerramientos opacos son estándar. Y la cubierta y los pisos de chapa rizada también son soluciones puestas en el mercado previamente a la concepción de este sistema concreto.

Lo mismo ocurre con el sistema TEST, pero aumentando el grado de compatibilidad de los componentes que utiliza el sistema (distintos modelos de paneles y carpinterías para los cerramientos). Y la estructura del 226×226x226 también está prevista para admitir distintas clases de paneles y cerramientos.
$\mathrm{Al}$ proyecto INVISO y la GBH les ocurre lo mismo (los pilares de "la Casa del Hombre" y de la GBH son los mismos, y los nudos de esta última admiten mayores posibilidades de enganche y combinatoria), por tanto se inscriben en el ámbito de la industrialización-abierta, que al emplear construcción ligera y montaje en seco, además es flexible.

Sin embargo, las piezas de la Dymaxion se fabrican a propósito para este sistema, no admiten las de otro, y solo admite algunos cambios en las divisiones interiores. Algo parecido le ocurre a la Abitazióni Evolutiva, pero valiéndose del montaje en construcción-ligera, el arquitecto prevé algunas opciones de instalación en entrepisos y carpintería exterior que le confieren al sistema suficiente flexibilidad para admitir diferentes clases de familia en su interior.

\subsection{Gestión del proceso}

La industrialización-abierta permite la fabricación de piezas y componentes al margen de la promoción directa de la edificación (sean viviendas o no). En el sector de las naves industriales o torres de oficinas, contratistas y promotores se encuentran más familiarizados con esta clase de mercado y recursos. Arquitectos e ingenieros saben generar sistemas estándar a partir de componentes de catálogo, y las responsabilidades legales se siguen repartiendo como si se estuviese construyendo un edificio especifico y singular (pero mucho más rápida y automáticamente).

La compra directa de componentes homologados a través de catálogo, facilita su fácil montaje, y puede llegar a permitir su gestión al margen de un contratista centralizado (la garantía total del sistema se llega a conseguir a partir de las garantías parciales de sus componentes: subsistemas).

Esto hace que la flexibilidad ofrecida por el sistema pueda llegar a ser manejada directamente por el habitante: autoconstrucción y bricolaje. 

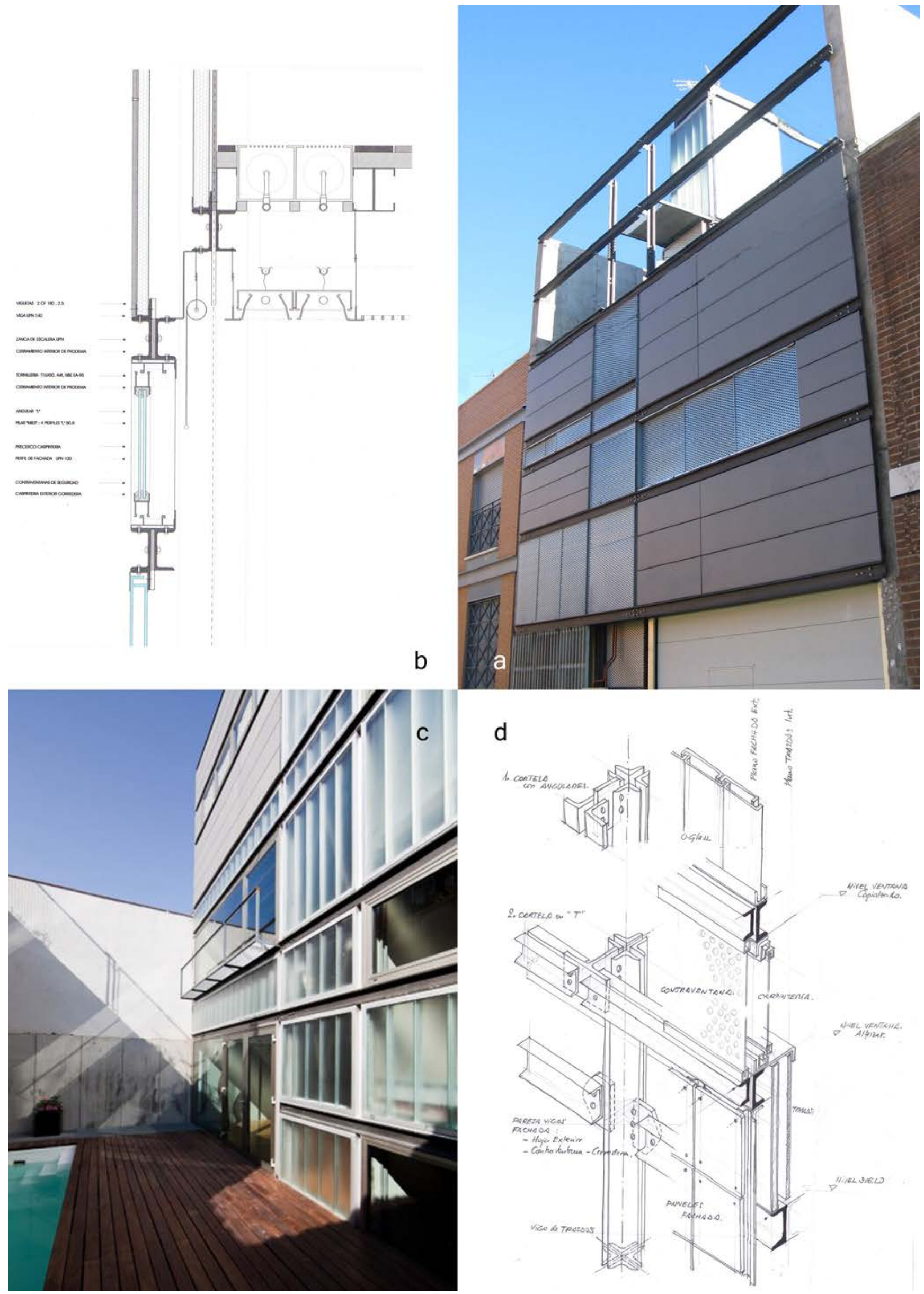

Figura 8. a) GBH: alzado Oeste; b) sección cerramiento Oeste; c) GBH alzado Este; d) montaje cerramiento Este. 


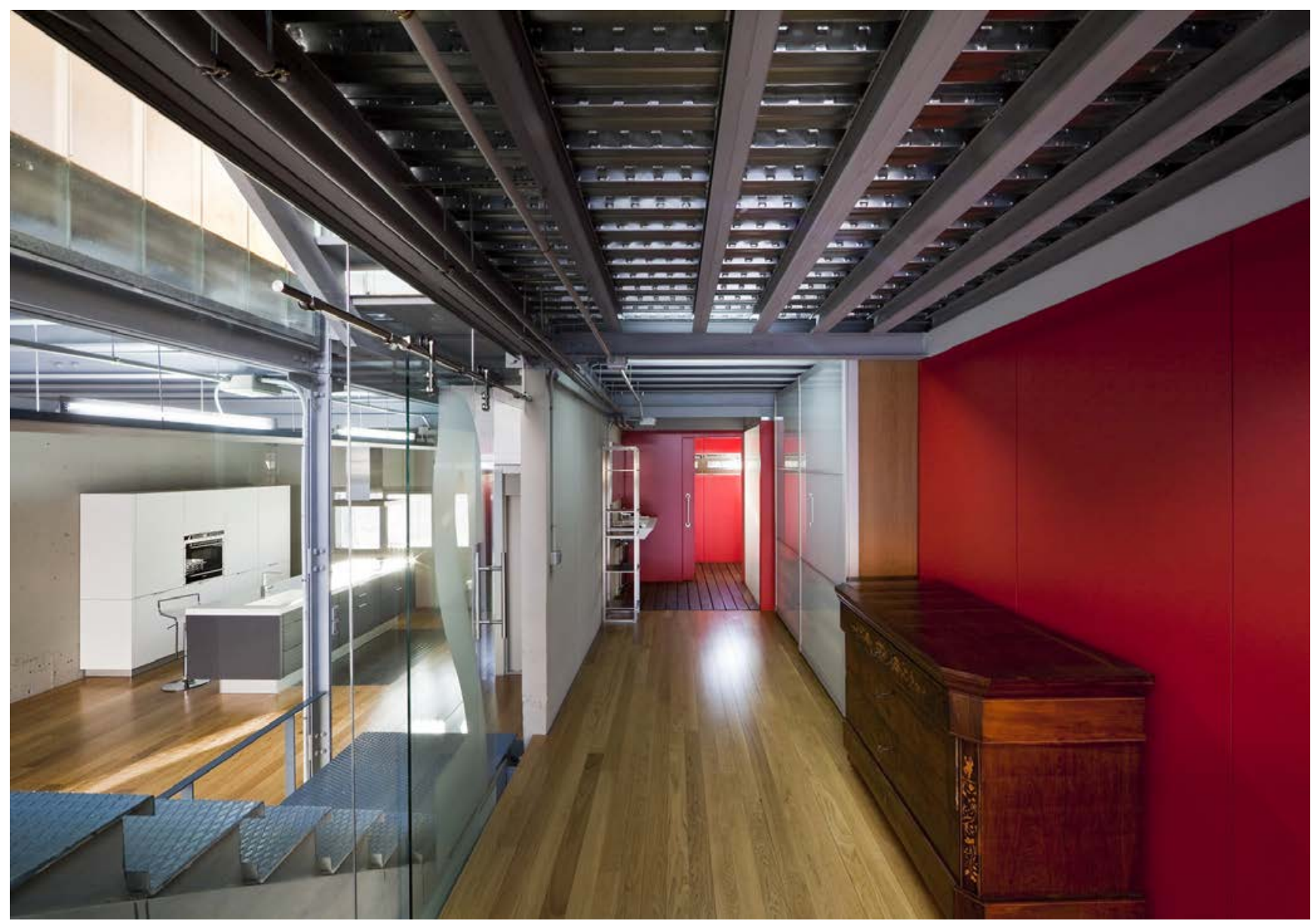

Figura 9. GBH interior con toda la construcción y las instalaciones "a la vista".

Esta distribución y venta directa de componentes (industriales) a su potencial usuario (con garantía de montaje a través de los instaladores de cada marca) hace cambiar las condiciones del mercado, de los profesionales afectados, y del control oficial de las construcciones (que pasa directamente a manos de las aseguradoras).

Todas estas transformaciones de mercado hacen que no sea fácil la puesta en práctica de estos sistemas basados en la industrialización-abierta, sino es a través del proceso convencional conocido, y de aquí se deriva el que tales sistemasabiertos "sin marca reconocida" se vean reflejados en pequeños edificios que solo aparentan su propia identidad, y sin embargo están esperando a ser rescatados por cualquier promotor innovador.

El "Revell Toy House", solo llego a extenderse hasta la tienda de H.Miller y no pasó de ser el juguete divulgativo que su promotor (el propio Miller) estaba dispuesto a pagar. Sin embargo, el TEST si tuvo varias aplicaciones en suelo Alemán. Y la Abitazioni Evolutiva quedo anclada a su punto de partida (la empresa fabricante de hormigón que hacia el encargo para su cooperativa de empleados quebró antes de llegar a darles cobijo).

Por otra parte, la aventura de Fuller y sus patentes no tiene parragón. Después de 20 años de regodeos y exposiciones entorno a la Dymaxion y sus readaptaciones, consigue poner en pie 2 prototipos de la Wichita House en 1946 con la Beech Aircraft Co., pero tras varias desavenencias en cuan- to a la explotación comercial, el proyecto se fue al traste (tampoco sus estructuras circulares de AL tuvieron la aceptación social que se esperaba, como le ocurrió a Eames con su sistema.)

El concurso INVISO se convocó en 2007 para que la EMVs pusiera en práctica el sistema en alguna de sus promociones, pero al poco explotó "la burbuja inmobiliaria" en la España de aquella década, y la GBH fue la única punta del iceberg que consiguió romper el cascarón.

Una punta que, contra viento y marea, consiguió ponerse en pie con una gestión al nivel en que la industrializaciónabierta aun se manifiesta en España. La principal dificultad se observó al inicio de la construcción, por su tamaño o por lo inesperado del planteamiento, ningún constructor al uso encajaba y hubo que realizar una gestión de la contratación por "paquetes", diferenciando por especialidades y marcas directamente desde el mercado. Lo que complicó por un lado la ejecución, pero puso a prueba la intercambiabilidad y autonomía de los componentes, según se había previsto.

\subsection{Componentes 3D para el S.21}

La Lloyd `s of London (Rogers+ partners 1983-87), supuso la confirmación de los componentes 3D incorporados a la mejor arquitectura del S XX (11). Previamente, el Nagaking Hotel (K. Kurokawa, 1972) y el Habitat de Montreal (M. Shadfi, 1967) (6) fueron importantes hitos en esta manera de pro- 
ducir, Pero hasta que en los 90 Toyota invade el mercado de vivienda unifamiliar en Japón sus condiciones de producción no fueron eficaces (12).

Así, los 3 ejemplos de S.3c nombrados al principio (ya en el S.21), son plenamente conscientes de la ventaja que la cadena de montaje ofrece a la arquitectura fabricada mediante componentes-3D (13).

El sistema INVISO plantea sus unidades 3D aplicadas solo a los espacios técnicos de sus viviendas. Pero el sistema de producción y situación de la GBH no permite su ensayo de manera económica y eficaz. La GBH sirve para comprobar los procesos de montaje y comportamiento de piezas en cuanto a estructura, cerramientos, circuitos de instalaciones, y divisiones interiores (componentes 1D y 2D), pero el ensayo de sus compactos de instalaciones tiene que esperar a mejor ocasión.

\section{ESTÁNDARES PARA UN S.3C}

Aunque es pronto para concretar un entorno regulatorio de aplicación a los S.3c se está acotando su territorio desde dos frentes diferentes. El referido a su sostenibilidad, y el que incide en sus procesos. En su actual nivel de desarrollo, ya hay comités internacionales de normalización trabajando en su definición:

- Criterios para la Sostenibilidad en el diseño de los proyectos y la construcción de sistemas, concretados en la norma en desarrollo ISO 20.887: Desing for Disassembly \& adaptability of buildings.
- Los procesos en la construcción por "paquetes de trabajo" que dentro de la gestión de proyectos industriales, incluso en la edificación, se conoce por WBS (Works Breakdown Structure): ISO 20511.

Ambas líneas de análisis y definición apoyan la promoción de la industrialización abierta, y tienen su aplicación directa sobre Sostenibilidad y los procesos de construcción en edificación.

\subsection{Sostenibilidad (arquitectura flexible y reciclable: adaptable)}

La Sostenibilidad aplicada a los sistemas constructivos se entiende actualmente según estas dos acepciones: "la adaptación”, referida a la flexibilidad en el uso de la edificación (que los edificios terminados puedan albergar más de un uso, con lo que su periodo de vida útil pueda alargarse, y no quedar sentenciados al poco de terminar su construcción); y "la desmontabilidad", con lo que la construcción puede ser restituida siempre a su inicio, sin grandes costes ni desperdicios. (desmantelamiento del sistema).

En la actualidad el comité de normalización ISO TC 59/SC 17 trabaja en torno al "desmontaje y adaptabilidad de edificios e ingeniería: DfD/A: DESIGN FOR DISASSEMBLY AND ADAPTABILITY, para la edición de la futura norma ISO 20887, y se estructura de la siguiente manera contemplando estos dos grupos con varias categorías cada uno:

- Adaptación, que comprende tres categorías relacionadas con la flexibilidad de uso:

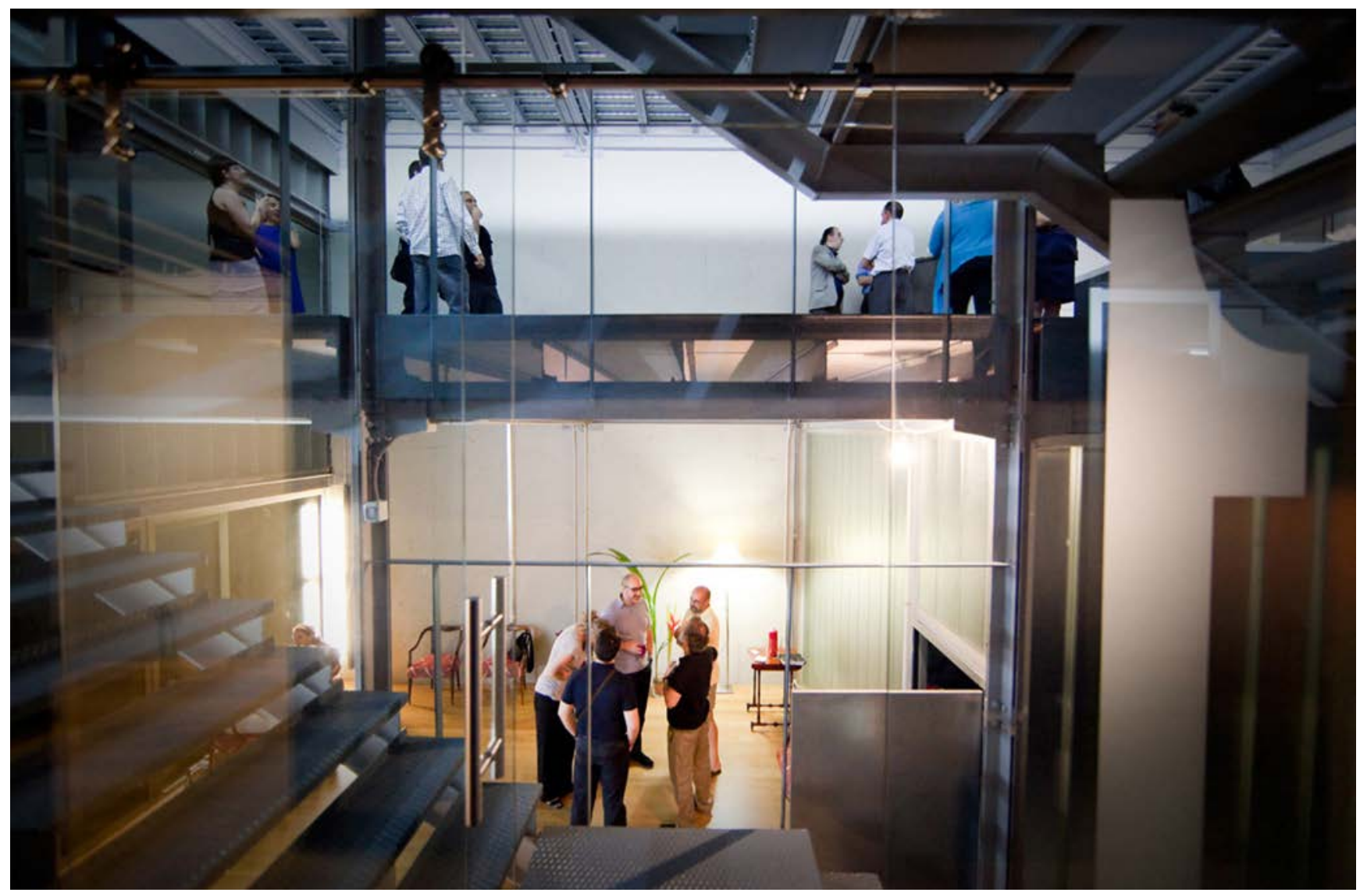

Figura 10. GBH: "interior fluido” desde la banda privada a los espacios comunes. 
Versatilidad (por su posibilidad de varios usos simultáneos), convertibilidad (que cambia el uso), expansión (que permite su ampliación).

- Desmontabilidad, que comprende: accesibilidad (facilidad de registro de los componentes) durabilidad (de mayor periodo de uso), conexiones expuestas y reversibles (facilidad para el intercambio de componentes, elementos o piezas), independencia (autonomía de funciones entre componentes), reciclado (posibilidad de vuelta a la reutilización de los materiales). Todo referido al montaje.

\subsection{Procesos de montaje (gestión y desarrollo)}

Si consideramos la edificación como un conjunto de procesos homologable a un montaje industrializado, nos podemos referir a la metodología del Project Management (PMg), que también está recogida en la normativa ISO (familia de normas ISO 21500).

El modelo del PMg es un sistema de organización por procesos, que para la construcción se contrapone al tradicional esquema de "promotor-arquitecto-constructor", facilitando los modos y conceptos de la ingeniería de sistemas. Así, se consigue la incorporación de componentes de forma más transparente y controlada, y los fabricantes pueden participar de manera más activa.

El WBS ("estructura desagregada de trabajos") es la herramienta dentro del PMg, que permite incorporar directamente los componentes de la construcción sin demasiada intermediación, donde los especialistas participan bajo la coordinación del $\mathrm{PMg}$, lo que beneficia el proceso y el resultado final. Consiste en una descomposición jerárquica de tareas, que subdivide el proyecto en unidades más elementales ("paquetes de trabajo") de menor complejidad y más fáciles de manejar, donde cada nivel descendente contempla una definición más detallada del trabajo a realizar.

El WBS también se encuentra actualmente en desarrollo su particular norma (ISO 21511) dentro del grupo de trabajo ISO TC 258, incluido en el grupo de la familia ISO 21.500.

Los paquetes de la WBS se definen por las siguientes características:

- Componentes de naturaleza común.

- Segregados por subproyectos que pueden ser realizados externamente.

- Identificados por componentes autónomos y de funcionalidad independiente.

(con todo ello se consigue una mayor flexibilidad de ejecución para el proyecto).

Para facilitar la configuración del WBS, debemos contemplar desde el diseño del sistema la composición de componentes en una jerarquía coordinada con las categorías mencionadas, algo que desemboca en el proyecto de subsistemas y grupos de componentes (estructura, piel, instalaciones ....), y paquetes de componentes en forma de complejos componentes $3 \mathrm{D}$.

La industrialización de la edificación, además de facilitar el uso de componentes, también debe automatizar los procesos en la construcción. Los principios de Sostenibilidad y el uso de herramientas de diseño como el "empaquetado" de componentes, son un camino.

\section{CONCLUSIONES}

\subsection{Comparación material entre sistema y prototipo (GBH-INVISO)}

La estructura de sistema y prototipo están en completa correspondencia: los perfiles, nudos, y placas de piso para el sistema INVISO se encuentran totalmente determinados en la casa GBH (en sus dimensiones y características físicas).

Se comprobaron la cantidad de variaciones necesarias en los nudos para una situación real, y como este se adaptaba a todas las necesidades reales (acoplamiento de perfiles de acero laminados: IPN, UPN, HEB, HEA,...LPN). También se comprueba la eficacia y seguridad del atornillamiento de alta resistencia en una estructura de esta escala y tamaño. Las velocidades de montaje también fueron notablemente rápidas (hoy día, la construcción de estructura metálica se orienta casi en exclusividad al procedimiento atornillado, y la soldadura queda relegada).

En cuanto a los cerramientos, se ensaya la fijación, estanqueidad y aislamiento de varios cerramientos planos de diferentes características: opacos, traslúcidos, practicables (componentes 2D) con los resultados marcados en proyecto (propiedades físicas), y eficacia y facilidad en su montaje (mayor rendimientos en m2/semana), y fijaciones seguras a base de montantes y travesaños de perfiles "deformados en frío", admitiendo tolerancias de $\mathrm{mm}$. Su suministro y gestión no supuso ninguna singularidad, y todos los materiales fueron adquiridos en el mercado de componentes de catálogo estandarizados al precio previsto y asequible (tecnología low-tech).

Los circuitos y canalización de instalaciones que figuran en el sistema INVISO, cobran realidad en el prototipo GBH y pasan de "la vertical principal" de fluidos (núcleo de instalaciones) hasta todos los capilares de cada habitación por todas las zonas y bandas que les corresponden sin estorbar ni interferir con el resto de sistemas: son independientes y registrables en todos sus puntos, contando con sus zonas de paso y reserva. Como ocurre con los sub-sistemas de estructura y cerramiento, todos los componentes necesarios para poner en práctica este sub-sistema se encuentran con total disponibilidad en el mercado de materiales estándar de la construcción: bandejas, ganchos, bridas, fijaciones, interruptores, luminarias...

A su vez, la experiencia de la GBH tiene su retorno para el INVISO de forma más abstracta y genérica, como se expone a continuación.

\subsection{Comparación sistémica entre sistema y prototipo (GBH-INVISO)}

Tanto del proyecto INVISO, como de la construcción de la $\mathrm{GBH}$, se pueden extraer 4 premisas de diseño preliminares, relacionadas con conceptos manejados en la Teoría General de Sistemas (aplicada al diseño en ingeniería de sistemas).

\section{Especialización de componentes:}

Subgrupos con función dedicada a una sola característica (soporte, aislamiento, conducción...). Los cerramientos de la GBH cumplen distintas funciones: visión, iluminación, opacidad, ... Y, por otra parte, en el INVISO se plantea un plano vertical de instalaciones, que recoge todos los aparatos y equipos de la instalación técnica que requiere la vivienda 
quedando separados de estas. Así, se dispone de un plano técnico que facilita tanto la instalación como el mantenimiento de sus funciones (Figura 5c).

Descomponibilidad del sistema:

Subgrupos del sistema que pueden ser diseñados y producidos por fabricantes y montadores independientes.

Cada subgrupo requiere un proceso de diseño aparte, con sus características y premisas diferenciadas. Los productos del mercado evolucionan diferenciadamente, integrándose posteriormente al sistema independientemente. Los pisos de la GBH son independientes de los cerramientos, y se pueden sustituir o desmontar sin condicionar el montaje de los subsistemas vecinos (Figura 8b). Igual ocurre con los componentes $3 \mathrm{D}$-comp del INVISO que se pueden enchufar/desenchufar al sistema general sin afectarlo en absoluto (Figura $5 \mathrm{c}$ ).

\section{Autonomía de componentes:}

Subgrupos autónomos diseñados para que su funcionamiento no interfiera con los colindantes. Se pueden montar o desmontar sin condicionar el funcionamiento del resto. Cada componente tiene sus características propias de funcionamiento: actividad, uso, registro, reposición..., sin repercutirse mutuamente con otros componentes. Las mamparas de la GBH montadas sobre perfiles directamente, permiten su función independiente de suelos y techos, lo que posibilita su desmontaje sin condicionar a otros. Igualmente, se configuran las unidades de habitación en el INVISO, sobre todo junto a cocinas y aseos, que quedan conectados a la vivienda de forma también autónoma.

Árbol de componentes (conjuntos, subconjuntos, ...):

Agrupación de componentes en paquetes, cada uno con sus propias leyes tanto de diseño como de funcionamiento y montaje, y que generan la complejidad del sistema. El conjunto replica nuevamente las categorías anteriores de especialización, autonomía y descomponibilidad, como nuevos componentes de grado superior identificables dentro del sistema. El sistema completo queda así dividido en estos paquetes (Figura 5c). y por tanto se reduce la complejidad de dos maneras:

- Se simplifican los vínculos entre componentes.

- Se facilita su control (al conocer en detalle los paquetes en sus características y relaciones internas).

La definición de componentes a partir de estas premisas, sus grados, su jerarquía, y su compatibilidad, da lugar a un orden orgánico donde la evolución del sistema es posible a favor de su flexibilidad de uso. Flexibilidad con ciertos grados de libertad, que se construye sobre una estructura conceptual y material totalmente orgánica.

\subsection{Los prototipos de la industrialización-abierta}

El ensayo del prototipo es crucial para cualquier producto industrial, pero su consecución es muy distinta si lo es para un sistema cerrado o abierto. Cuando el sistema es cerrado, todo queda bajo la misma patente, $\mathrm{y}$ al responder un solo fabricante de todos los componentes, es el propio fabricante quien se encarga de ensayar el prototipo y su homologación. El prototipo se desarrolla por partes o entero dentro de la propia factoría, y los laboratorios oficiales se encargan del resto.

Pero al referirnos a la industrialización abierta, y por no haber un solo fabricante que responda de todo el sistema, entonces suele ser el propio creador del Sistema de construcción por componentes compatibles (S.3c) quien debe enfrentarse a su puesta a punto, y esto sin tener aun a la vista empresa alguna dispuesta a promoverlo. En estos casos, el inventor del sistema suele ser un arquitecto, o equipo de profesionales (ingenieros de sistemas) que, en el mejor de los casos, se ven impelidos a convertirse en promotores del mismo. Y esto es lo que le da gran valor a todos los ejemplos que aquí se traen en donde, tras la inofensiva apariencia de una pequeña construcción individual, se esconde todo el potencial de un sistema constructivo que puede llegar a ver su desarrollo en el mercado industrial.

Estos modelos de procesos, con suficiente referencia internacional, mediante el Project Management, no están aún reconocidos en nuestra legislación ni en la Ley de Contratos del Estado; pero en su tendencia de aplicación profesional, se espera su incorporación a nivel oficial próximamente. Pese a ello, esto no impide el que se puedan construir colegios, hospitales, o viviendas a partir de S.3c, manteniendo la vía administrativa vigente: por ahora, sigue siendo responsabilidad del Arquitecto el que esta clase de procedimientos se pongan en práctica.

\section{REFERENCIAS}

(1) Torres, J. (2004). Le Corbusier: visiones de la técnica en cinco tiempos. p. 70, 87, 186, Madrid: F. Caja de Arquitectos.

(2) Gorman, M.J. (2005). Buckmister Fuller desing for mobility, p. 23-39. Milan: Skiraeditore.

(3) Eames, C. (1949). CSH for 1949. Arts \&Architecture, (dic-1949):41-54.

(4) Reyes, J.M. (1985). S3C, prototipos de vivienda construidos por componentes compatibles. Informes de la Construcción, 37(373): 5-16, doi: https://doi.org/10.3989/ic.1985.v37.i373.1822

(5) Estratto da "Lezioni di Design": Renzo Piano -La Casa Evolutiva (1978). https://www.youtube.com/watch?v=iPJojHF-hRE\&fs $=1 \&$ hl $=$ es_ES

(6) Broadhurts, R. (2008). Home delivery, p. 214-223, 144-147, 124-127, NYC: MoMA, Brikhauser.

(7) Almagro, M., Vega S. (2011). Solar Decthlon Europe 2010. p. 138-147. Madrid: ten act1on

(8) http://hicarquitectura.com/2012/o7/herreros-arquitectos-casa-garoza/

(9) Reyes J.M. (2010). 4 Juegos para 4 avatares, p. 16-31, Madrid: Mairea-libros.

(10) Oteiza, I., Queipo de Llano, J. (2009). Concurso de ideas INVISO. Informes de la Construcción, 60(512): 73-75, doi: https://doi.org/10.3989/ic.08.042

(11) Powell, K. (1999). Pioneering British High-Tech. Londres: Phaidon.

(12) Catálogo Toyota-home (1996). Tokyo: Toyota Co.

(13) Reyes, J.M. (2011). Sistemas de construcción Standard. Madrid: Esc. de la Edificación (UNED). 